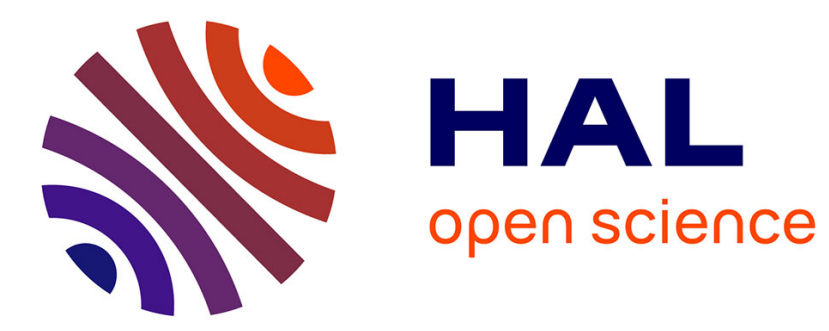

\title{
Niveau de vie et déplacements dans les métropoles vietnamiennes : Hô Chi Minh ville et Hanoi
}

\author{
Patrick Gubry, Linh Lê Hô Phong
}

\section{To cite this version:}

Patrick Gubry, Linh Lê Hô Phong. Niveau de vie et déplacements dans les métropoles vietnamiennes :

Hô Chi Minh ville et Hanoi. Revue Tiers Monde, 2010, 201, pp.107-129. ird-00473626

\section{HAL Id: ird-00473626 \\ https://hal.ird.fr/ird-00473626}

Submitted on 15 Apr 2010

HAL is a multi-disciplinary open access archive for the deposit and dissemination of scientific research documents, whether they are published or not. The documents may come from teaching and research institutions in France or abroad, or from public or private research centers.
L'archive ouverte pluridisciplinaire HAL, est destinée au dépôt et à la diffusion de documents scientifiques de niveau recherche, publiés ou non, émanant des établissements d'enseignement et de recherche français ou étrangers, des laboratoires publics ou privés. 
[Fichier PDF auteur - article publié dans la Revue Tiers Monde, 2010, n² 201, p. 107-129.]

\title{
Niveau de vie et déplacements dans les métropoles vietnamiennes : Hô Chi Minh Ville et Hanoi
}

\author{
Patrick Gubry \\ IRD, UMR 201 Université Paris 1 \\ gubry@ird.fr \\ Lê Hô Phong Linh \\ Ho Chi Minh City Institute for Development Studies (HIDS), Hô Chi Minh Ville \\ lhplinh@yahoo.com
}

Le Viêt-nam est entré dans une phase d'urbanisation rapide suite à la libéralisation progressive de l'économie mise en œuvre à partir de 1986. Hô Chi Minh Ville, la « capitale économique » (environ 5,9 millions d'urbains en 2009) et Hanoi, la capitale politique (environ 2,6 millions d'urbains) sont les deux métropoles qui concentrent la majeure partie de la croissance urbaine et des investissements (Central Population and Housing Census Steering Committee, 2009) $)^{1}$.

Dans un contexte où l'accroissement naturel de la population est devenu très faible en ville, par suite de la transition démographique, avec la baisse de la fécondité, la croissance migratoire est le facteur essentiel de la croissance urbaine. La migration rurale-urbaine est impulsée par la concentration des investissements et l'accroissement des disparités économiques entre la ville et la campagne, au bénéfice de la première ; elle est facilitée par la rémanence d'une forte proportion de population rurale (70,4\% dans l'ensemble du pays), réservoir important de migrants potentiels.

La croissance démographique et la métropolisation génèrent une extension géographique de l'espace urbain, par suite de l'afflux de migrants en ville (souvent en zone périphérique) et des migrations intra-urbaines (surtout en direction de la périphérie également). Cette extension élargit l'espace de vie et accroît les mobilités temporaires auxquelles les villes sont mal préparées. Cependant, dans les deux villes, l'augmentation de la population soulève des problèmes cruciaux dans le processus de développement concernant les infrastructures, l'environnement et bientôt l'emploi.

Dans un contexte de « gentrification » du centre-ville et d'accroissement des disparités socio-économiques à l'intérieur même du périmètre urbain, il y a lieu de s'interroger sur les conséquences de cette évolution sur les déplacements respectifs des "riches» et des « pauvres », car les déplacements - « obligés » pour le travail et l'école, ou « de convenance »

\footnotetext{
${ }^{1} \mathrm{Au}$ Viêt-nam, les limites administratives des grandes agglomérations incluent une vaste zone rurale : Hô Chi Minh Ville compte ainsi 7,1 M d'habitants et Hanoi 6,4 M, en incluant les parties rurales. Une étude précise de la population urbaine de ces agglomérations demanderait de retirer du total la population urbaine des petites localités disjointes du centre $(\mathrm{Cu}$ Chi et Cân Gio à Hô Chi Minh Ville, Son Tây à Hanoi) et à l'inverse d'ajouter à l'agglomération de Hô Chi Minh Ville les zones urbaines contiguës des provinces de Binh Duong et de Dông Nai (ville de Biên Hoa).
} 
pour les loisirs- constituent une part majeure de la qualité de vie. La comparaison entre les deux métropoles est à cet égard riche d'enseignements.

\section{Une politique de « déconcentration » de la population}

Les migrations intra-urbaines, qui tendent à augmenter les mobilités temporaires en termes de distance et de temps de déplacement sont déterminées par l'évolution du prix du foncier et par les politiques publiques.

L'augmentation de la pression démographique au centre-ville, associée à une disparité socio-économique croissante et à la montée d'une classe moyenne, entraîne une explosion du prix du foncier. Appartenant à l'État depuis la collectivisation, le foncier et l'immobilier connaissent une privatisation ou une semi-privatisation croissantes: les occupants d'une parcelle résidentielle se voient dotés d'un titre qui leur permet maintenant de disposer de leurs biens sans limitation de durée, d'en vendre le droit d'usage et de le transmettre à leurs héritiers, même si l'État reste officiellement le seul «propriétaire » du sol ${ }^{2}$. Dans ce contexte, les ménages les plus modestes n'ont pas les moyens d'entretenir leur patrimoine au centreville, tandis qu'il devient rentable de le vendre à des ménages aisés pour acheter un bien plus spacieux et plus confortable dans un arrondissement périphérique. À cela s'ajoute la construction de villas en périphérie par les ménages aisés en vue de spéculation ou dans la perspective d'y résider ultérieurement, quand les infrastructures se seront développées.

Au niveau des politiques publiques en matière d'urbanisme, on retiendra les quatre préoccupations suivantes :

- «L'assainissement» du centre : il s'agit essentiellement de détruire l'habitat insalubre le long des canaux (arroyos à Hô Chi Minh Ville) et de délocaliser les habitants vers la périphérie (cas du projet Nhiêu Loc-Thi Nghê par exemple).

- La réduction des fortes densités et de la promiscuité : certains secteurs centraux connaissent des densités records : plus de 115000 habitants au $\mathrm{km}^{2}$ dans le phuong $1 \mathrm{du} 3^{\mathrm{e}}$ arrondissement de Hô Chi Minh Ville en 1999, plus de 92000 habitants au $\mathrm{km}^{2}$ dans le phuong Hang Gai à Hanoi $^{3}$. L'évolution engagée permet d'ores et déjà de relever une diminution de la population en chiffres absolus dans certains arrondissements durant la période intercensitaire 1989-1999 ( $5^{\mathrm{e}}$ arrondissement de Hô Chi Minh Ville).

- La volonté « d'internationaliser » et de «tertiariser» le centre, avec l'objectif de faire ainsi des deux grandes métropoles des « villes mondiales » [Global cities/ Thành phố toàn cầu].

- Les programmes de construction en périphérie : la ceinture périphérique de chacune des deux métropoles est par excellence la zone de développement des programmes de constructions à usage résidentiel. C'est le cas par exemple à Hô Chi Minh Ville de la zone de Phu My Hung ("Saigon South», dans le $7^{\mathrm{e}}$ arrondissement), de celle de Thu Thiêm ( $2^{\mathrm{e}}$ arrondissement), ou à Hanoi du projet Linh Dam dans l'arrondissement de Thanh Tri, du complexe Ciputra dans l'arrondissement de Tây Hô, des zones urbaines nouvelles de Câu Giây, de Bac An Khan et Duong Noi (dans le grand ouest de Hanoi). Pour saisir l'ampleur de ces programmes, il faut signaler que 240 projets de «Zones urbaines nouvelles » (Khu do thi $m o i$ ) ont été validés pour Hanoi en octobre 2009, destinés à s'ajouter à la dizaine de projets

\footnotetext{
${ }^{2}$ Les subtilités de l'évolution du foncier à Hanoi sont parfaitement mises en lumière dans Pandolfi, 2001.

${ }^{3}$ Le phuong est l'unité administrative immédiatement inférieure à l'arrondissement ; en milieu rural (y compris dans les arrondissements ruraux inclus dans les limites administratives des grandes villes) ces unités sont appelées $x a$.
} 
existants... Bien entendu, ces programmes s'accompagnent d'une reclassification de l'usage du sol qui le rend constructible.

Ces différents facteurs tendent à accroître les migrations du centre vers la périphérie et à densifier les zones périphériques. Qu'en est-il dans les faits?

\section{Le « desserrement » de la population en direction de la périphérie}

Le recensement est un outil irremplaçable pour l'étude des migrations (changement de résidence), au niveau géographique le plus fin, puisqu'il est par vocation exhaustif aux erreurs de collecte près ${ }^{4}$. À partir d'un recensement, il est plus facile d'étudier l'immigration dans une zone donnée (qui concentre les immigrants), que l'émigration à partir de la même zone (qui disperse les émigrants entre toutes les unités administratives du pays, voire tous les pays du monde).

À Hô Chi Minh Ville, si l'on prend la population âgée de cinq ans ou plus en 1999, 83,7 \% résidaient déjà dans le même arrondissement en 1994, cinq ans auparavant, 6,7 \% résidaient dans un autre arrondissement de $\mathrm{HCMV}, 9,3 \%$ résidaient dans une autre province et $0,2 \%$ à l'étranger.

À Hanoi, les chiffres correspondants sont les suivants : 87,7\% des habitants résidaient dans le même arrondissement de Hanoi en 1999 qu'en 1994, 4,0 \% résidaient dans un autre arrondissement de Hanoi, $8,0 \%$ résidaient dans une autre province et $0,3 \%$ à l'étranger. Globalement, la population apparaît un peu plus « stable » à Hanoi, dont le taux de croissance serait légèrement inférieur à celui de Hô Chi Minh Ville.

Selon les conditions locales dans les divers pays et les spécificités des villes, les immigrants dans les grandes agglomérations se dirigent prioritairement soit vers le centre, soit vers la périphérie. Au Viêt-nam, les immigrants se dirigent très clairement de manière préférentielle vers les arrondissements situés en périphérie immédiate du centre, aussi bien à Hô Chi Minh Ville qu'à Hanoi.

Dans l'une et l'autre ville les mouvements ne sont significatifs que dans les arrondissements centraux et dans les arrondissements immédiatement périphériques. Les zones rurales ne sont guère touchées entre 1994 et 1999, quand on examine les déplacements de la population âgée de 5 ans ou plus ${ }^{5}$. Les mouvements dessinés par les soldes migratoires sont clairement centrifuges, du centre vers la périphérie, conduisant à un "desserrement » de la population. Ces mouvements sont plus diversifiés à Hanoi.

\footnotetext{
${ }^{4}$ Ce desserrement a été analysé en détail dans Gubry \& al., 2008, chapitre 2, p. 53-62. Les données équivalentes au recensement d'avril 2009 ne sont pas encore disponibles en fin 2009.

${ }^{5}$ Voir cartes correspondantes dans Gubry \& al., 2008, p. 55-56. http://horizon.documentation.ird.fr/exl-doc/pleins_textes/divers09-01/010043543.pdf
} 


\section{Une enquête spécifique sur les mobilités intra-urbaines}

Les déplacements temporaires peuvent être étudiés à travers diverses approches : analyse des visiteurs et des absents dans le recensement lorsque ces données sont disponibles, enquête sur la circulation, enquête spécifique auprès des ménages... Nous avons choisi de réaliser une enquête par sondage représentative auprès des ménages, approche qui n'avait encore jamais été tentée au Viêt-nam sur ce sujet, et de mettre en œuvre un plan de sondage original. Cette enquête a été réalisée en mars-avril 2003 dans le cadre du Programme de Recherche Urbaine pour le Développement (PRUD).

Le projet de recherche sur les mobilités intra-urbaines à Hô Chi Minh Ville et Hanoi est l'un des 32 projets réalisés dans le cadre du PRUD financés par le Ministère français des Affaires Étrangères et gérés par l'ISTED et le GEMDEV (2002-2004). Il s'agit d'un projet en coopération entre l'Institut de Recherche pour le Développement (IRD), Paris ; l'Institut de recherche économique de Hô Chi Minh Ville (IER); le Centre de population, Université nationale d'économie, Hanoi.

Les travaux préliminaires ont conduit à élaborer un plan de sondage à deux degrés. Au premier degré, on a tiré un échantillon d'îlots ou tô dân phô ${ }^{6}$, profitant du fait que l'espace est "îloté » à un niveau très fin et que le responsable d'îlot connaît en principe les chefs de ménage de son îlot. Au second degré, on a tiré un échantillon de ménages sur la liste des chefs de ménage préalablement établie dans les îlots sélectionnés. À chacun des degrés, l'échantillon est en pratique géographiquement stratifié dans la mesure où les îlots d'un côté, les ménages de l'autre se suivent sensiblement sur les listes selon l'ordre de proximité géographique. Le but était d'arriver à un échantillon de 2000 ménages à Hô Chi Minh Ville et 1500 ménages à Hanoi.

Ce plan de sondage est novateur, dans la mesure où jusqu'à présent on utilisait généralement les phuong/xa au premier degré, ce qui génère un effet de grappe considérable (ces unités ont de 3000 à 4000 ménages chacun), ce qui n'est pas le cas des îlots (de l'ordre de 40 à 100 ménages). En outre, l'exhaustivité est meilleure dans la mesure où les listes des ménages sont établies indépendamment des listes existantes auprès du responsable d'îlot qui ne comprennent que les personnes ayant fait une démarche pour régulariser leur situation en ville.

Un programme informatique spécifique a été élaboré par un spécialiste de l'INSEE pour d'une part tirer les îlots sur la liste des îlots de chaque ville, d'autre part les ménages sur la liste des ménages établie lors de la première étape dans les îlots sélectionnés ${ }^{7}$.

Finalement, l'échantillon comprend 2000 ménages et 8623 habitants à Hô Chi Minh Ville, 1500 ménages et 5972 habitants à Hanoi.

\footnotetext{
${ }^{6}$ En zone périphérique, les îlots portent les noms de tô nhân dân à Hô Chi Minh Ville et thôn ou xom à Hanoi.

${ }^{7}$ La méthodologie détaillée de cette enquête, la seule de ce type à ce jour sur les mobilités intraurbaines, est exposée dans Gubry \& al., 2008, chapitre 1, p. 19-50.
} 


\section{Variation spatiale du niveau de vie des ménages}

\section{Utilisation d'un indice de richesse}

Plusieurs méthodes permettent de mesurer le statut économique relatif des ménages. Dans notre enquête, nous avons proscrit les questions directes sur les revenus et les dépenses des ménages à cause de leur faible qualité dans une enquête ponctuelle à cause des défaillances de la mémoire des enquêtés et d'une volonté fréquente de sous-estimer les revenus. Cependant, nous avons recueilli des informations détaillées sur les caractéristiques du logement, sa localisation, le type d'occupation, les services de base et les équipements durables à la disposition des ménages. En plus, notre objectif n'est pas de mesurer directement le statut économique des ménages, en valeur absolue, mais les différences de niveau de vie entre des groupes de ménages.

Des études spécifiques montrent que l'indice de richesse est une méthode appropriée à cette fin (Filmer \& Pritchett, 1998 ; Howe \& al., 2008 ; Rutstein \& Johnson, 2004 ; Vyas \& Kumaranayake, 2006). Nous avons ainsi utilisé 38 indicateurs pour calculer un indice de richesse.

Dans cette étude, on a utilisé le logiciel Stata (version 8) et l'indice est calculé comme suit avec la méthode d'analyse en composantes principales :

- 1) Vérification de l'ordre relatif des variables ordinales.

- 2) Standardisation des indicateurs (pour que les variables suivent une distribution normale).

- 3) Utilisation de la méthode d'analyse en composantes principales pour calculer le poids des indicateurs. La première composante principale est choisie pour représenter la richesse du ménage. Le poids de chaque indicateur est la valeur de la covariance de la première composante de la matrice de covariance.

- 4) Calcul de la valeur de l'indice pour les ménages. Pour chaque ménage, la valeur des indicateurs est multipliée par les poids correspondants et l'indice de richesse du ménage est la somme de ces valeurs.

L'hypothèse a été retenue que chaque membre d'un même ménage a le même niveau de richesse, ainsi tous les membres du ménage ont le même indice de richesse. Tous les individus de l'ensemble des deux villes ont été ensuite triés dans l'ordre croissant de richesse et ont été divisés en trois groupes égaux, les «pauvres» (niveau de richesse « faible»), les « moyens » (niveau de richesse « moyen ») et les « riches » (niveau de richesse « élevé »). 
Un processus de « gentrification » du centre

On assiste à une concentration progressive des ménages aisés dans les arrondissements centraux des deux villes (tableau 1). La population des arrondissements centraux représente $67,9 \%$ à Hô Chi Minh Ville mais 86,2\% des ménages aisés résident dans ces arrondissements; les chiffres correspondants pour Hanoi sont respectivement $46,6 \%$ et $67,9 \%{ }^{8}$.

Tableau 1 : Répartition de la population selon le niveau de richesse, la zone de résidence et la ville $(\%)$

\begin{tabular}{|c|l|c|c|c|c|}
\hline Ville & $\begin{array}{l}\text { Niveau } \\
\text { de richesse }\end{array}$ & $\begin{array}{c}\text { Arrondissements } \\
\text { centraux }\end{array}$ & $\begin{array}{c}\text { Arrondissements } \\
\text { périphériques }\end{array}$ & $\begin{array}{c}\text { Arrondissements } \\
\text { ruraux }\end{array}$ & Total \\
\hline \multirow{4}{*}{ HCMV } & Faible & 50,4 & 21,7 & 27,9 & 100 \\
\cline { 2 - 6 } & Moyen & 67,3 & 18,8 & 13,9 & 100 \\
\cline { 2 - 6 } & Élevé & 86,2 & 8,9 & 4,9 & 100 \\
\cline { 2 - 6 } & Ensemble & 67,9 & 16,6 & 15,4 & 100 \\
\hline \multirow{4}{*}{ Hanoi } & Faible & 27,4 & 9,5 & 63,1 & 100 \\
\cline { 2 - 6 } & Moyen & 51,5 & 19,5 & 29,0 & 100 \\
\cline { 2 - 6 } & Élevé & 67,3 & 22,7 & 10,0 & 100 \\
\cline { 2 - 6 } & Ensemble & 46,6 & 16,4 & 37,0 & 100 \\
\hline
\end{tabular}

Source : Enquête PRUD, 2003 ; données pondérées

À l'inverse, les ménages avec un niveau de vie relativement faible sont clairement concentrés dans les arrondissements ruraux des deux villes.

Le processus de "gentrification » du centre-ville ressort clairement de l'examen du niveau de vie selon l'arrondissement (figure 1).

\footnotetext{
${ }^{8}$ À l'exclusion des arrondissements de $\mathrm{Cu}$ Chi et Cân Gio à Hô Chi Minh Ville, et de Soc Son à Hanoi, presque purement ruraux. Comme on l'a souligné, le maintien d'une catégorie d'arrondissements classés comme " ruraux » (huyên) à côté des arrondissements " urbains » (quân) ne doit pas étonner, car les limites administratives des deux métropoles incluent une vaste zone rurale regroupant, suite aux modifications administratives de 2006 et surtout du $1^{\text {er }}$ août 2008 , $16,8 \%$ de la population à Hô Chi Minh Ville et 59,2 \% de la population à Hanoi, au recensement de 2009.
} 


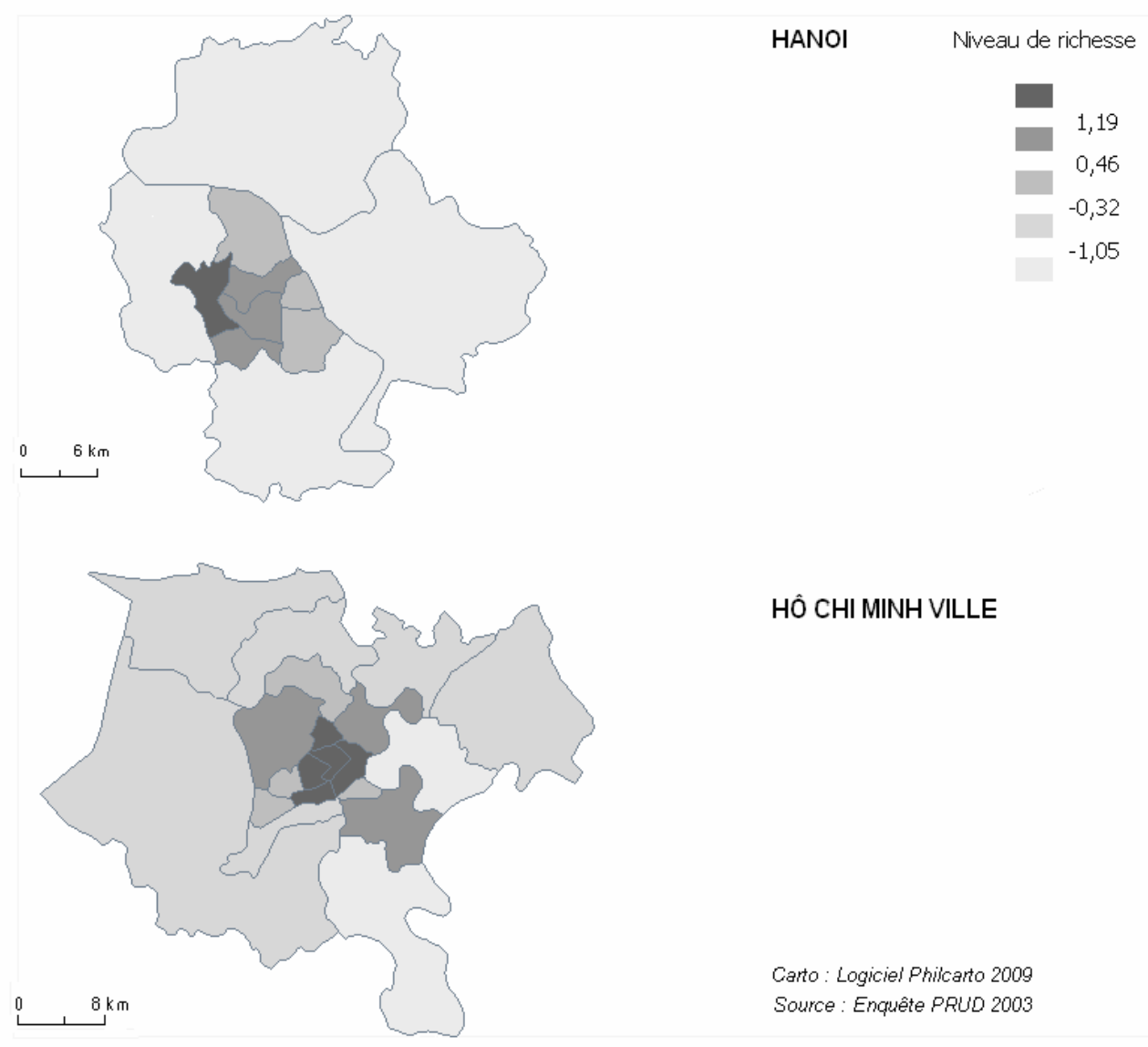

Figure 1 : Indice de richesse selon l'arrondissement et la ville

À Hô Chi Minh Ville, la «richesse» est ainsi concentrée dans les $1^{\mathrm{e}}, 3^{\mathrm{e}}, 10^{\mathrm{e}}$ arrondissements, dans l'arrondissement de Phu Nhuân et dans le $5^{\mathrm{e}}$ arrondissement (Cho Lon), dont la population est souvent d'origine chinoise. Les arrondissements ruraux, souvent agricoles, sont clairement les plus pauvres; entre les deux, dans les arrondissements périphériques, une population à niveau de vie modeste se mélange à des classes moyennes aisées qui ont accès aux nouveaux programmes immobiliers.

À Hanoi, les arrondissements les plus «riches » sont également les arrondissements centraux, surtout l'arrondissement de Ba Dinh, mais également l'arrondissement de Câu Giai, à l'écart du centre, qui possède de nombreux programmes immobiliers modernes, ainsi que le nouveau « centre moderne » de la capitale, My Dinh (qui s'étend également en partie sur Tu Liêm). Les arrondissements ruraux, de Tu Liêm à Gia Lâm, y compris toute la rive gauche du fleuve Rouge, encore largement agricole, sont les plus «pauvres».

\section{Le rôle ambivalent du permis de résidence}

Au Viêt-nam, l'ensemble du territoire national est subdivisé en îlots, que ce soit à la ville (tô dân phô) ou à la campagne (tô nhân dân), c'est « l'îlotage ». L'îlot est une unité de police inférieure à la plus petite unité administrative (phuong ou $x a$ ). Chaque citoyen est enregistré dans un îlot donné. Un changement de résidence implique théoriquement une autorisation au niveau de l'îlot de départ et une autorisation au niveau de l'îlot d'arrivée. 
La résidence est enregistrée au niveau du phuong (arrondissements urbains) ou du $x a$ (arrondissements ruraux) sur un livret de résidence (hô khâu). Il y a quatre types de permis de résidence ${ }^{9}$ :

- KT1 : résidents permanents dans un îlot du phuong qui ont un permis de résidence permanent y afférant ;

- KT2: $\quad$ - soit résidents permanents dans un îlot du phuong qui ont un permis relatif à un autre phuong de la même province ;

- soit personnes qui ont un permis relatif à un îlot du phuong (qui y sont enregistrées sur un registre spécial), mais qui résident dans un autre phuong dans la même province ;

- KT3 : résidents temporaires de longue durée dans un îlot du phuong (ayant de facto vocation à demeurer sur place) ;

- KT4 : résidents temporaires de courte durée dans un îlot du phuong (ayant vocation à repartir) ou visiteurs.

Dans le passé, la possession d'un permis de résidence permanent (KT1) était indispensable pour résider en ville dans la mesure où il donnait lieu à la distribution des bons de rationnement; cela éliminait d'emblée les migrations spontanées, mais les bons de rationnement n'existent plus depuis longtemps. Progressivement, le rôle du permis de résidence s'est trouvé changé et le permis de résidence a été considéré comme la pièce maîtresse de la politique migratoire du Viêt-nam. Cependant, les enquêtes récentes montrent que l'absence de permis de résidence n'empêche plus personne de migrer et le permis de résidence ne peut plus être considéré comme un élément d'une politique migratoire ou d'une politique urbaine (Cf. par exemple, Gubry \& al., 2002). Pourtant, le contrôle résidentiel est maintenu et reste effectif malgré les apparences. De nos jours, l'argent remplace parfois avantageusement la disposition d'un permis de résidence en règle : il s'agit en somme pour un individu de soupeser les coûts respectifs de la régularisation et du paiement d'une amende...

Cependant, il ne convient pas pour autant de considérer le permis de résidence comme inutile. Au contraire, de nombreux actes de la vie quotidienne sont facilités par la possession d'un permis permanent. Cela va des plus importants, comme l'immatriculation foncière, l'achat d'une maison, l'exercice d'un emploi dans le secteur public, l'inscription d'un enfant dans une école renommée, l'obtention des meilleurs soins à l'hôpital... jusqu'à des formalités plus banales comme l'immatriculation d'une motocyclette ou l'abonnement à une ligne téléphonique fixe...

Rares sont les études qui se sont penchées sur ce système (Hardy, 2001 ; VeT \& al., 2005). Une des conclusions est que le système d'enregistrement résidentiel reste une source d'inégalités à l'intérieur de la ville au détriment des plus pauvres. C'est notamment ce qui a conduit les autorités à largement assouplir le système à partir de 2006, en rendant plus facile l'obtention d'un permis de résidence permanent, sans pour autant éliminer tous les obstacles.

Dans ce contexte, il y a lieu de s'interroger sur les conséquences de l'enregistrement résidentiel sur les déplacements en relation avec le niveau de vie. La répartition de la population ayant un enregistrement permanent varie selon l'arrondissement (figure 2), mais la situation est différente dans les deux villes.

\footnotetext{
${ }^{9}$ KT de kiêm tra $=$ contrôler.
} 
À Hô Chi Minh Ville, les habitants des arrondissements centraux, à population relativement stable, ont souvent un enregistrement permanent, mais également ceux de certains arrondissements éloignés tels que le $9^{\mathrm{e}}$ arrondissement (qui a une population encore rurale, associée à des travailleurs de grandes entreprises modernes qui sont généralement enregistrés) ou le $7^{\mathrm{e}}$ arrondissement (avec de nombreux lotissements modernes). En revanche, les habitants du reste de la zone périphérique, même dans sa partie rurale, sont moins souvent enregistrés, pour des raisons qui sont mal discernables, si ce n'est que ces zones contiennent une forte proportion de migrants.

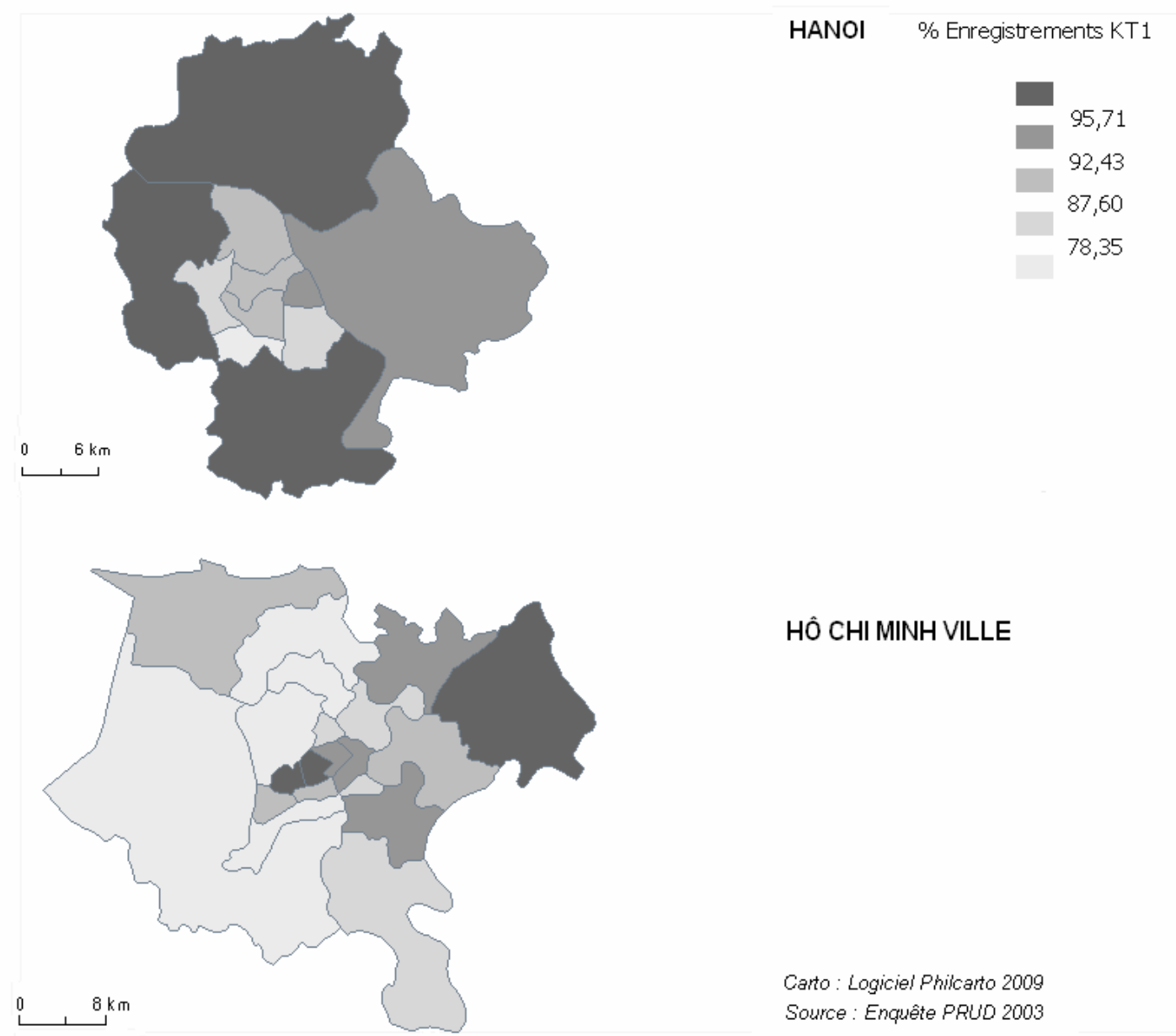

Figure 2 : Proportion de la population ayant un permis de résidence permanent (KT1) selon l'arrondissement de résidence et la ville $(\%)$

À Hanoi, la situation est sans équivoque : les habitants des arrondissements ruraux périphériques sont très majoritairement enregistrés, vivant et travaillant souvent sur place depuis plusieurs générations.

Il faut remarquer que les ménages les plus modestes habitent précisément dans ces zones rurales. Il s'en suit que les « riches » sont $85,3 \%$ à posséder un permis de résidence permanent à Hô Chi Minh Ville (73,2 \% pour les « pauvres »), contre 82,2 \% à Hanoi $(94,5 \%$ pour les «pauvres »). L'effet du statut de résident permanent est ainsi occulté par celui de la zone d'habitation et de l'activité. 


\section{Des motifs de déplacements différenciés}

Pour déterminer les déplacements temporaires, on a relevé les déplacements hors du logement effectués le jour précédant le passage de l'enquêteur. Un des principaux résultats a été de constater que les déplacements pour le travail restent importants tous les jours de la semaine, y compris pendant le week-end (Gubry \& al., 2008). Qu'en est-il des raisons de déplacements selon le niveau de vie?

La fréquence des sorties est peu différente entre Hanoi et Hô Chi Minh Ville (tableau 2). Les riches ont plus de raisons de sortir que les pauvres, mais les différences entre les groupes sont faibles.

Tableau 2 : Répartition des personnes de 13 ans ou plus selon les raisons de sortie du logement, le niveau de richesse et la ville (\%)

\begin{tabular}{|l|r|r|r|r|r|r|r|r|}
\hline \multirow{2}{*}{$\begin{array}{c}\text { Raisons de sortie } \\
\text { du logement }\end{array}$} & \multicolumn{4}{|c|}{ Hô Chi Minh Ville } & \multicolumn{5}{c|}{ Hanoi } \\
\cline { 2 - 10 } & Pauvres & Moyens & Riches & Ens, & Pauvres & Moyens & Riches & Ens, \\
\hline Sorti & 83,7 & 86,5 & 88,8 & 86,4 & 82,0 & 86,4 & 89,5 & 85,6 \\
\hline Pas sorti & 16,3 & 13,5 & 11,2 & 13,6 & 18,0 & 13,6 & 10,5 & 14,4 \\
\hline 1, Travail & 38,7 & 37,4 & 38,1 & 38,0 & 35,5 & 36,2 & 38,1 & 36,5 \\
\hline 2, École/formation & 8,3 & 10,9 & 14,4 & 11,2 & 12,6 & 12,8 & 12,7 & 12,7 \\
\hline $\begin{array}{l}\text { 3, Amener les enfants } \\
\text { à l'école }\end{array}$ & 3,7 & 5,2 & 7,2 & 5,4 & 2,1 & 2,5 & 3,3 & 2,6 \\
\hline 4, Courses & 16,5 & 18,1 & 19,3 & 18,0 & 16,3 & 19,6 & 18,2 & 17,8 \\
\hline 5, Visite à la famille & 3,2 & 2,9 & 2,5 & 2,9 & 3,2 & 3,2 & 4,3 & 3,5 \\
\hline 6, Visite aux amis & 4,4 & 3,9 & 3,9 & 4,1 & 4,0 & 5,1 & 6,6 & 5,1 \\
\hline 7, Dispensaire/hôpital & 0,5 & 1,0 & 0,6 & 0,7 & 0,3 & 0,4 & 0,5 & 0,4 \\
\hline 8, Loisirs & 1,9 & 2,8 & 5,0 & 3,2 & 2,9 & 5,3 & 5,3 & 4,3 \\
\hline 9, Divers & 0,5 & 0,6 & 0,8 & 0,6 & 0,3 & 0,4 & 0,6 & 0,4 \\
\hline
\end{tabular}

Source : Enquête PRUD, 2003 ; données pondérées

En ce qui concerne les raisons de sortie, à Hô Chi Minh Ville, les pauvres sont plus nombreux à être sortis pour le travail, au contraire de Hanoi.

À Hô Chi Minh Ville, les riches sont plus nombreux à avoir accompagné leurs enfants à l'école, à la fois parce qu'ils disposent de meilleurs moyens de transports et parce que leurs enfants fréquentent en moyenne des établissements plus éloignés de leur domicile, qu'ils ont pu choisir à cause de leur bonne réputation. Les différences sont peu marquées à Hanoi.

Les riches s'adonnent aussi plus fréquemment aux loisirs, parce qu'ils ont les moyens nécessaires et que les pauvres doivent consacrer plus de temps au travail pour gagner leur vie.

Une explication importante de ces différences est aussi que les riches ont de bons moyens de transport: la presque totalité des membres des ménages riches dans les villes dispose d'une motocyclette dans le ménage tandis que seulement 79,6\% des membres des ménages pauvres à Hô Chi Minh Ville et 59,5\% à Hanoi en disposent. 


\section{Résidence et lieu de travail}

Les arrondissements centraux concentrent logiquement les emplois dans les deux villes (tableau 3). Une proportion de $92,7 \%$ des actifs occupés à Hô Chi Minh Ville et de $86,5 \%$ à Hanoi y trouve leur emploi. Ce sont les habitants des arrondissements périphériques qui ont le plus de déplacements à faire pour aller travailler. En revanche, dans les arrondissements ruraux, nombreux sont ceux qui travaillent près de leur domicile ; c'est le cas notamment des agriculteurs qui vivent sur leur exploitation.

\section{Tableau 3 : Répartition de la population active occupée selon la zone d'habitation, la zone de travail et la ville $(\%)$}

\begin{tabular}{|c|c|c|c|c|c|c|}
\hline \multirow[b]{2}{*}{ Ville } & \multirow[b]{2}{*}{ Zone d'habitation } & \multicolumn{3}{|c|}{ Zone de travail } & \multirow[b]{2}{*}{ Total } & \multirow[b]{2}{*}{ 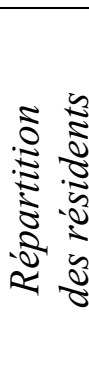 } \\
\hline & & 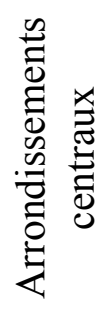 & 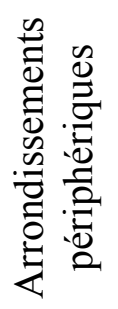 & 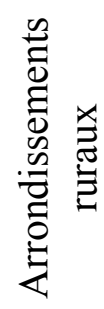 & & \\
\hline \multirow{4}{*}{ HCMV } & $\begin{array}{l}\text { Arrondissements } \\
\text { centraux }\end{array}$ & 92,7 & 4,0 & 3,3 & 100 & 67,9 \\
\hline & $\begin{array}{l}\text { Arrondissements } \\
\text { périphériques }\end{array}$ & 19,5 & 77,7 & 2,8 & 100 & 16,6 \\
\hline & $\begin{array}{l}\text { Arrondissements } \\
\text { ruraux }\end{array}$ & 15,2 & 5,2 & 79,7 & 100 & 15,4 \\
\hline & Ensemble/Total & 69,1 & 16,1 & 14,8 & 100 & 100 \\
\hline \multirow{4}{*}{ Hanoi } & $\begin{array}{l}\text { Arrondissements } \\
\text { centraux }\end{array}$ & 86,5 & 7,4 & 6,1 & 100 & 46,6 \\
\hline & $\begin{array}{l}\text { Arrondissements } \\
\text { périphériques }\end{array}$ & 40,1 & 52,7 & 7,2 & 100 & 16,4 \\
\hline & $\begin{array}{l}\text { Arrondissements } \\
\text { ruraux }\end{array}$ & 10,5 & 4,2 & 85,3 & 100 & 37,0 \\
\hline & Ensemble/Total & 47,1 & 13,4 & 39,5 & 100 & 100 \\
\hline
\end{tabular}

Source : Enquête PRUD, 2003 ; données pondérées

La population active occupée se répartit très différemment selon le niveau de richesse et la zone de travail (tableau 4). Dans les deux villes, la population aisée travaille très majoritairement au centre-ville, alors que la population à niveau de vie modeste travaille plutôt dans les arrondissements ruraux. Cela correspond aux revenus des types d'activités que ces zones peuvent offrir et peut être rapproché de l'indice de richesse par arrondissement (figure 1).

Les localisations respectives du domicile et du lieu de travail permettent de confronter la distance et la durée du trajet (déclarés par les intéressés) au niveau de richesse (tableau 5). Distance et durée moyenne restent somme toute raisonnables pour des grandes métropoles : 4,9 km et 15,7 mn à Hô Chi Minh Ville ; 3,7 km et 14,5 mn à Hanoi. 
Tableau 4 : Répartition de la population active occupée selon le niveau de richesse et la zone de travail $(\%)$

\begin{tabular}{|c|l|c|c|c|c|}
\hline \multirow{3}{*}{ Ville } & $\begin{array}{c}\text { Niveau } \\
\text { de richesse }\end{array}$ & $\begin{array}{c}\text { Arrondissements } \\
\text { centraux }\end{array}$ & $\begin{array}{c}\text { Arrondissements } \\
\text { périphériques }\end{array}$ & $\begin{array}{c}\text { Arrondissements } \\
\text { ruraux }\end{array}$ & Total \\
\hline \multirow{5}{*}{ HCMV } & Faible & 54,6 & 20,2 & 25,2 & 100 \\
\cline { 2 - 6 } & Moyen & 66,3 & 19,1 & 14,6 & 100 \\
\cline { 2 - 6 } & Élevé & 86,6 & 8,5 & 4,9 & 100 \\
\cline { 2 - 6 } & Ensemble & 69,1 & 16,1 & 14,8 & 100 \\
\hline \multirow{4}{*}{ Hanoi } & Faible & 25,2 & 10,4 & 64,4 & 100 \\
\cline { 2 - 6 } & Moyen & 54,3 & 15,4 & 30,3 & 100 \\
\cline { 2 - 6 } & Élevé & 71,7 & 16,0 & 12,2 & 100 \\
\cline { 2 - 6 } & Ensemble & 47,1 & 13,4 & 39,5 & 100 \\
\hline
\end{tabular}

Source : Enquête PRUD, 2003 ; données pondérées

Tableau 5 : Répartition de la population active occupée selon le niveau de richesse, la distance moyenne pour aller au travail $(\mathrm{km})$, le temps moyen $(\mathrm{mn})$, la zone d'habitation et la ville

\begin{tabular}{|c|c|c|c|c|c|c|c|}
\hline \multirow[b]{2}{*}{ Ville } & \multirow[b]{2}{*}{$\begin{array}{l}\text { Niveau } \\
\text { de richesse }\end{array}$} & \multirow[b]{2}{*}{$\begin{array}{c}\text { Distance }(\mathrm{km}) / \\
\text { Durée }(\mathrm{mn})\end{array}$} & \multicolumn{3}{|c|}{ Zone d'habitation } & \multirow[b]{2}{*}{ 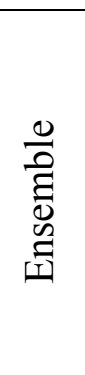 } & \multirow[b]{2}{*}{$\begin{array}{l}\text { Vitesse } \\
\text { moyenne } \\
(\mathrm{km} / \mathrm{mn})\end{array}$} \\
\hline & & & 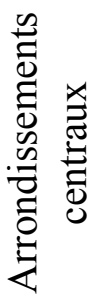 & 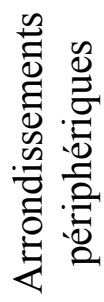 & 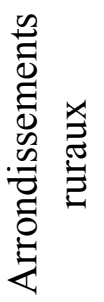 & & \\
\hline \multirow{8}{*}{ HCMV } & \multirow{2}{*}{ Faible } & Distance moyenne & 4,0 & 5,2 & 4,3 & 4,4 & \multirow{2}{*}{0,27} \\
\hline & & Durée moyenne & 15,5 & 17,2 & 17,0 & 16,3 & \\
\hline & \multirow{2}{*}{ Moyen } & Distance moyenne & 5,2 & 4,4 & 4,1 & 4,9 & \multirow{2}{*}{0,32} \\
\hline & & Durée moyenne & 16,0 & 14,5 & 12,7 & 15,3 & \\
\hline & \multirow{2}{*}{ Élevé } & Distance moyenne & 5,2 & 6,3 & 8,0 & 5,4 & \multirow{2}{*}{0,34} \\
\hline & & Durée moyenne & 15,3 & 16,0 & 21,8 & 15,7 & \\
\hline & \multirow{2}{*}{ Ensemble } & Distance moyenne & 4,9 & 5,0 & 4,6 & 4,9 & \multirow{2}{*}{0,31} \\
\hline & & Durée moyenne & 15,6 & 15,8 & 16,1 & 15,7 & \\
\hline \multirow{8}{*}{ Hanoi } & \multirow{2}{*}{ Faible } & Distance moyenne & 3,7 & 3,1 & 2,7 & 3,0 & \multirow{2}{*}{0,21} \\
\hline & & Durée moyenne & 14,0 & 13,9 & 13,9 & 13,9 & \\
\hline & \multirow{2}{*}{ Moyen } & Distance moyenne & 3,7 & 5,0 & 3,6 & 4,0 & \multirow{2}{*}{0,28} \\
\hline & & Durée moyenne & 13,8 & 16,9 & 13,2 & 14,2 & \\
\hline & \multirow{2}{*}{ Élevé } & Distance moyenne & 4,4 & 4,7 & 5,2 & 4,6 & \multirow{2}{*}{0,29} \\
\hline & & Durée moyenne & 14,9 & 16,6 & 17,7 & 15,6 & \\
\hline & \multirow{2}{*}{ Ensemble } & Distance moyenne & 4,1 & 4,4 & 3,1 & 3,7 & \multirow{2}{*}{0,26} \\
\hline & & Durée moyenne & 14,3 & 16,1 & 14,0 & 14,5 & \\
\hline
\end{tabular}

Source : Enquête PRUD, 2003 ; données pondérées

La distance et la durée du trajet domicile-travail sont logiquement les plus élevées pour les habitants des arrondissements périphériques. À Hanoi, il s'agit de la proche 
périphérie ; à Hô Chi Minh Ville, la situation est plus complexe et dépend en grande partie des lotissements récents implantés dans des zones périphériques qui n'offrent pas encore suffisamment d'emplois (figure 3).
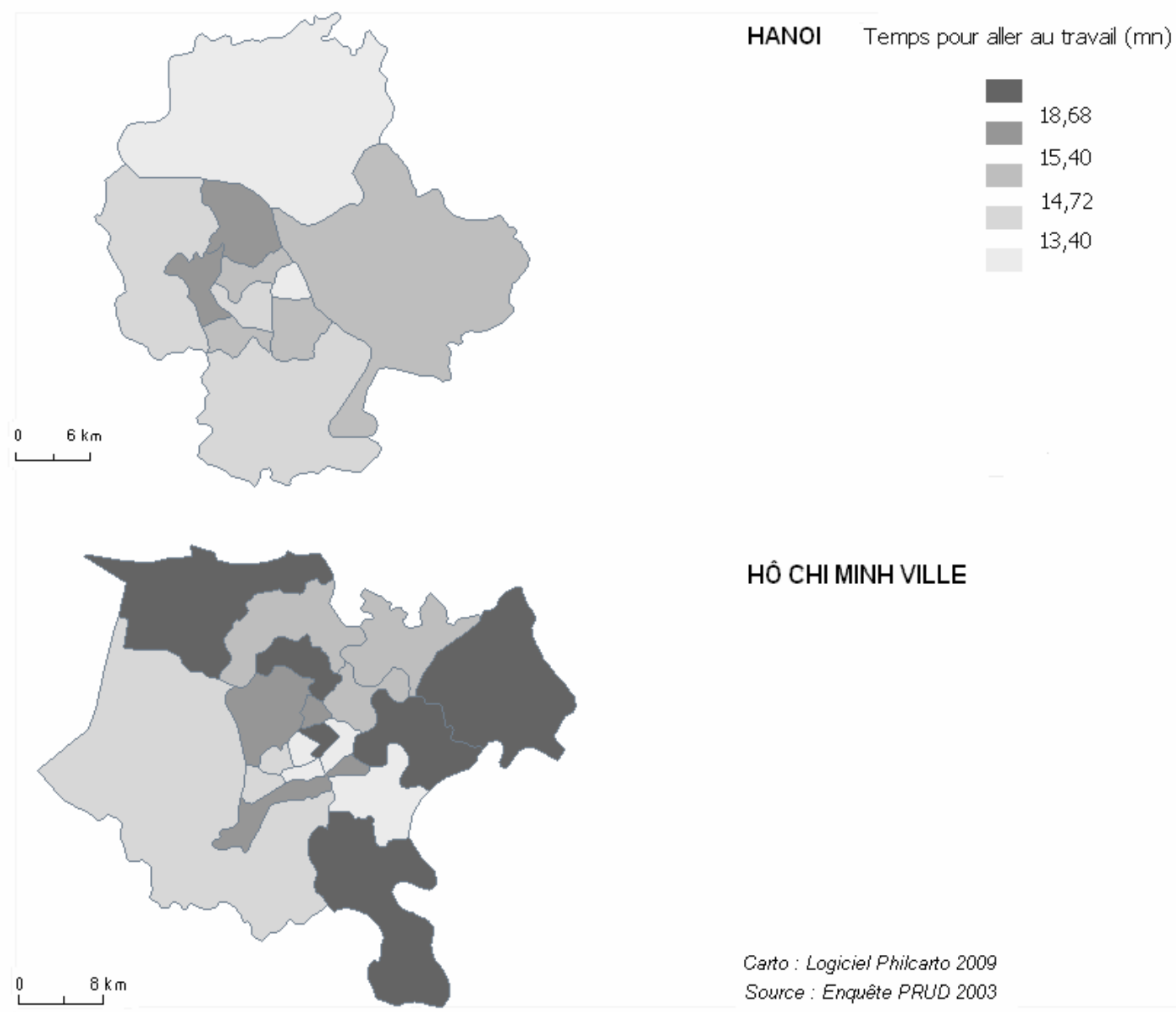

HÔ CHI MINH VILLE

Carto : Logiciel Philcarto 2009

Source : Enquête PRUD 2003

\section{Figure 3 : Temps mis pour aller au travail selon l'arrondissement de résidence et la ville (mn)}

Les riches ont une distance plus grande à parcourir que les pauvres. Leurs moyens de transport leur permettent de choisir et de conserver un lieu de travail de manière relativement indépendante de sa localisation, en diminuant le temps du trajet. Au total, on observe que la vitesse moyenne du trajet domicile-travail augmente avec le niveau de richesse.

\section{Modes de transport}

La motocyclette est le moyen de transport de loin le plus fréquent pour effectuer le trajet domicile-travail : 74,1\% des actifs occupés à Hô Chi Minh Ville et 51,7\% à Hanoi y ont recours (tableau 6 et figure 4). L'usage de la motocyclette est directement proportionnel au niveau de richesse et varie en sens contraire de celui de la bicyclette qui lui est concurrente, les autres moyens de transport, notamment collectifs, restant très faibles. La marche à pieds est beaucoup plus répandue à Hanoi où les distances sont plus faibles, indépendamment du niveau de vie. 
Tableau 6 : Répartition de la population active occupée selon le niveau de richesse, le moyen de transport pour aller au travail et la ville (\%)

\begin{tabular}{|c|l|r|r|c|c|c|c|c|}
\hline \multirow{2}{*}{ Ville } & $\begin{array}{c}\text { Niveau } \\
\text { de richesse }\end{array}$ & \multicolumn{5}{|c|}{ Moyen de transport pour aller au travail } & \multirow{2}{*}{ Total } \\
\cline { 2 - 9 } & À pieds & Bicyclette & Motocyclette & Voiture & Bus & Autre & \\
\hline \multirow{4}{*}{ HCMV } & Faible & 15,3 & 23,5 & 54,5 & 0,4 & 1,7 & 4,5 & 100 \\
\cline { 2 - 9 } & Moyen & 7,4 & 9,9 & 79,1 & 1,5 & 0,3 & 1,8 & 100 \\
\cline { 2 - 9 } & Élevé & 3,1 & 3,6 & 88,8 & 1,0 & 0,7 & 2,8 & 100 \\
\cline { 2 - 9 } & Ensemble & 8,7 & 12,3 & 74,1 & 1,0 & 0,9 & 3,0 & 100 \\
\hline \multirow{4}{*}{ Hanoi } & Faible & 41,3 & 28,5 & 27,1 & 0,3 & 1,4 & 1,3 & 100 \\
\cline { 2 - 9 } & Moyen & 19,6 & 18,2 & 58,0 & 0,9 & 2,2 & 1,1 & 100 \\
\cline { 2 - 9 } & Élevé & 5,5 & 7,4 & 80,1 & 2,1 & 2,5 & 2,3 & 100 \\
\cline { 2 - 9 } & Ensemble & 24,5 & 19,3 & 51,7 & 1,0 & 2,0 & 1,6 & 100 \\
\hline
\end{tabular}

Source : Enquête PRUD, 2003 ; données pondérées

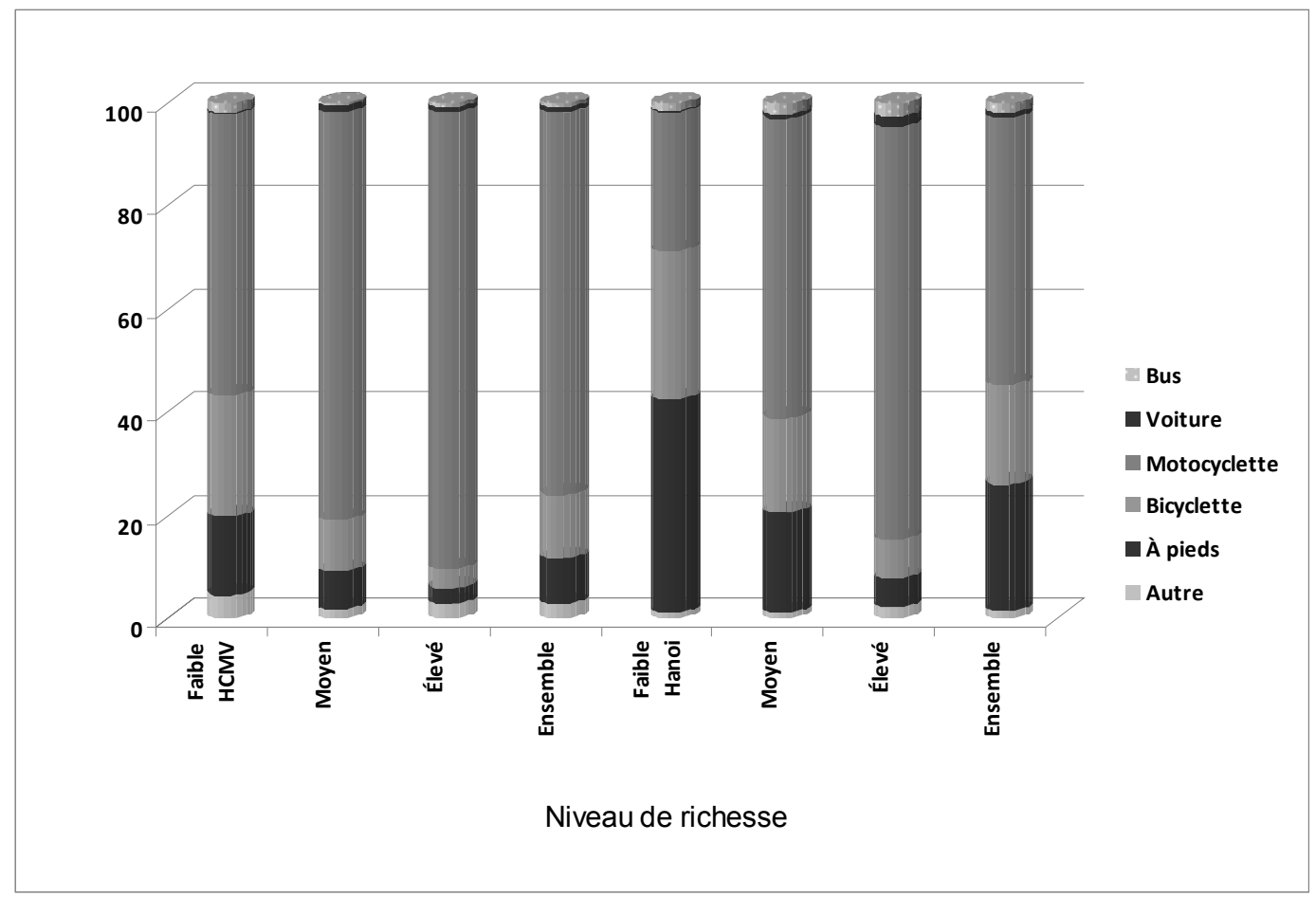

Figure 4 : Répartition de la population active occupée selon le moyen de transport pour aller au travail, le niveau de richesse de leur ménage et la ville (\%)

\section{Résidence et école}

Les établissements scolaires sont plus concentrés dans les arrondissements centraux que les emplois à Hô Chi Minh Ville, au contraire de Hanoi, où les arrondissements ruraux semblent relativement bien pourvus (tableau 7). Nous examinons ici la zone d'étude au niveau global; une distinction entre écoles primaires, collèges, lycées et universités montrerait évidemment une concentration accrue des établissements dans le centre au fur et à mesure de l'élévation du niveau d'enseignement. 
Tableau 7 : Répartition des élèves selon la zone d'habitation, la zone d'étude et la ville $(\%)$

\begin{tabular}{|c|c|c|c|c|c|c|}
\hline \multirow[b]{2}{*}{ Ville } & \multirow[b]{2}{*}{$\begin{array}{c}\text { Zone } \\
\text { d'habitation }\end{array}$} & \multicolumn{4}{|c|}{ Zone d'étude } & \multirow[b]{2}{*}{ 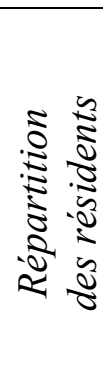 } \\
\hline & & 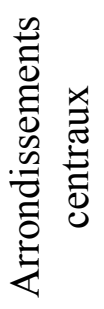 & 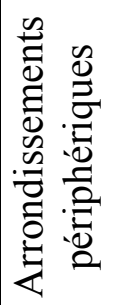 & 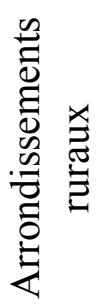 & Total & \\
\hline \multirow{4}{*}{ HCMV } & $\begin{array}{l}\text { Arrondissements } \\
\text { centraux }\end{array}$ & 98,3 & 1,5 & 0,2 & 100 & 67,9 \\
\hline & $\begin{array}{l}\text { Arrondissements } \\
\text { périphériques }\end{array}$ & 13,7 & 82,2 & 4,1 & 100 & 16,6 \\
\hline & $\begin{array}{l}\text { Arrondissements } \\
\text { ruraux }\end{array}$ & 18,8 & 2,9 & 78,3 & 100 & 15,4 \\
\hline & Ensemble/Total & 73,1 & 14,8 & 12,1 & 100 & 100 \\
\hline \multirow{4}{*}{ Hanoi } & $\begin{array}{l}\text { Arrondissements } \\
\text { centraux }\end{array}$ & 89,8 & 9,1 & 1,1 & 100 & 46,6 \\
\hline & $\begin{array}{l}\text { Arrondissements } \\
\text { périphériques }\end{array}$ & 32,1 & 62,4 & 5,5 & 100 & 16,4 \\
\hline & $\begin{array}{l}\text { Arrondissements } \\
\text { ruraux }\end{array}$ & 9,0 & 6,4 & 84,6 & 100 & 37,0 \\
\hline & Ensemble/Total & 45,7 & 18,0 & 36,2 & 100 & 100 \\
\hline
\end{tabular}

Source : Enquête PRUD, 2003 ; données pondérées

Les familles aisées scolarisent leurs enfants très majoritairement dans un établissement localisé au centre-ville (tableau 8). Ils ont les moyens de choisir un établissement renommé, de payer éventuellement des frais plus élevés et d'accompagner leurs enfants vers une école plus éloignée du domicile.

Tableau 8 : Répartition des élèves selon le niveau de richesse de leur ménage, la zone d'étude et la ville (\%)

\begin{tabular}{|c|l|c|c|c|c|}
\hline \multirow{3}{*}{ Ville } & \multirow{2}{*}{$\begin{array}{c}\text { Niveau } \\
\text { de richesse }\end{array}$} & $\begin{array}{c}\text { Arrondissements } \\
\text { centraux }\end{array}$ & $\begin{array}{c}\text { Arrondissements } \\
\text { périphériques }\end{array}$ & $\begin{array}{c}\text { Arrondissements } \\
\text { ruraux }\end{array}$ & Total \\
\hline \multirow{4}{*}{ HCMV } & Faible & 57,5 & 19,2 & 23,3 & 100 \\
\cline { 2 - 6 } & Moyen & 67,6 & 19,2 & 13,2 & 100 \\
\cline { 2 - 6 } & Élevé & 90,4 & 6,9 & 2,7 & 100 \\
\cline { 2 - 6 } & Ensemble & 73,1 & 14,8 & 12,1 & 100 \\
\hline \multirow{4}{*}{ Hanoi } & Faible & 25,0 & 12,5 & 62,5 & 100 \\
\cline { 2 - 6 } & Moyen & 54,9 & 20,9 & 24,2 & 100 \\
\cline { 2 - 6 } & Élevé & 67,0 & 23,3 & 9,7 & 100 \\
\cline { 2 - 6 } & Ensemble & 45,7 & 18,0 & 36,2 & 100 \\
\hline
\end{tabular}

Source: Enquête PRUD, 2003 ; données pondérées 
$\mathrm{Au}$ contraire, les familles modestes sont souvent contraintes d'inscrire leurs enfants dans l'école correspondant à la carte scolaire de leur domicile, surtout si elles ne disposent pas d'un enregistrement résidentiel permanent en ville.

Logiquement, la distance pour aller à l'école est plus courte que pour aller au travail (tableau 9). Dans les deux villes, la distance pour aller à l'école augmente avec le niveau de richesse par suite du choix d'une meilleure école pour leurs enfants par les plus fortunés ; ce phénomène est plus marqué à Hô Chi Minh Ville.

\section{Tableau 9 : Distance moyenne $(\mathrm{km})$ et durée moyenne $(\mathrm{mn})$ mis par les élèves pour aller à l'école selon le niveau de richesse de leur ménage, la zone d'habitation et la ville}

\begin{tabular}{|c|c|c|c|c|c|c|c|}
\hline \multirow[b]{2}{*}{ Ville } & \multirow[b]{2}{*}{$\begin{array}{l}\text { Niveau } \\
\text { de richesse }\end{array}$} & \multirow[b]{2}{*}{$\begin{array}{c}\text { Distance }(\mathrm{km}) / \\
\text { Durée }(\mathrm{mn})\end{array}$} & \multicolumn{4}{|c|}{ Zone d'habitation } & \multirow[b]{2}{*}{$\begin{array}{l}\text { Vitesse } \\
\text { moyenne } \\
(\mathrm{km} / \mathrm{mn})\end{array}$} \\
\hline & & & 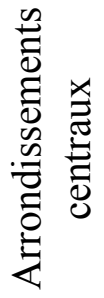 & 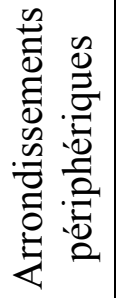 & 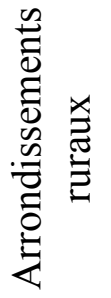 & 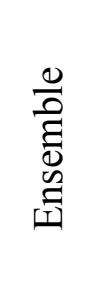 & \\
\hline \multirow{8}{*}{ HCMV } & \multirow{2}{*}{ Faible } & Distance moyenne & 2,6 & 2,9 & 3,2 & 2,8 & \multirow{2}{*}{0,19} \\
\hline & & Durée moyenne & 14,6 & 14,9 & 13,5 & 14,4 & \\
\hline & \multirow{2}{*}{ Moyen } & Distance moyenne & 3,0 & 3,1 & 3,9 & 3,2 & \multirow{2}{*}{0,23} \\
\hline & & Durée moyenne & 13,1 & 13,6 & 17,1 & 13,8 & \\
\hline & \multirow{2}{*}{ Élevé } & Distance moyenne & 3,8 & 5,4 & 5,6 & 4,0 & \multirow{2}{*}{0,26} \\
\hline & & Durée moyenne & 14,8 & 16,3 & 19,4 & 15,2 & \\
\hline & \multirow{2}{*}{ Ensemble } & Distance moyenne & 3,3 & 3,5 & 3,8 & 3,4 & \multirow{2}{*}{0,23} \\
\hline & & Durée moyenne & 14,2 & 14,5 & 15,6 & 14,4 & \\
\hline \multirow{8}{*}{ Hanoi } & \multirow{2}{*}{ Faible } & Distance moyenne & 2,6 & 4,2 & 3,3 & 3,2 & \multirow{2}{*}{0,21} \\
\hline & & Durée moyenne & 13,3 & 16,5 & 15,8 & 15,4 & \\
\hline & \multirow{2}{*}{ Moyen } & Distance moyenne & 2,5 & 4,1 & 3,8 & 3,3 & \multirow{2}{*}{0,22} \\
\hline & & Durée moyenne & 11,9 & 15,8 & 17,8 & 14,6 & \\
\hline & \multirow{2}{*}{ Élevé } & Distance moyenne & 3,4 & 3,8 & 4,2 & 3,6 & \multirow{2}{*}{0,24} \\
\hline & & Durée moyenne & 14,5 & 15,7 & 18,2 & 15,2 & \\
\hline & \multirow{2}{*}{ Ensemble } & Distance moyenne & 2,9 & 4,0 & 3,4 & 3,3 & \multirow{2}{*}{0,22} \\
\hline & & Durée moyenne & 13,4 & 16,0 & 16,4 & 15,1 & \\
\hline
\end{tabular}

Source : Enquête PRUD, 2003 ; données pondérées

On retrouve l'augmentation avec le niveau de richesse de la vitesse moyenne du trajet pour aller à l'école, comme pour aller au travail ; elle dépend ici du véhicule utilisé par les parents pour accompagner leurs enfants à l'école pour les petites classes, et du moyen de transport personnel (bicyclette ou motocyclette) utilisé par les plus grands.

La figure 5, concernant l'école secondaire, fait ressortir les arrondissements souséquipés en infrastructures scolaires, ceux où le trajet pour aller à l'école est le plus long. Il s'agit surtout des arrondissements situés en périphérie, qu'ils soient urbains ou ruraux : toute la rive gauche du fleuve Rouge à Hanoi ; l'arrondissement de Hoc Môn à Hô Chi Minh Ville. 
Mais certains arrondissements centraux figurent aussi dans cette catégorie: les arrondissements de Ba Dinh et de Tây Hô à Hanoi, qui sont des cas particuliers dans la mesure où la population qui y réside, très aisée en moyenne, peut envoyer ses enfants dans une école éloignée mais réputée, ainsi que le $4^{\mathrm{e}}$ arrondissement de Hô Chi Minh Ville, qui est sans doute sous-équipé en infrastructures scolaires.
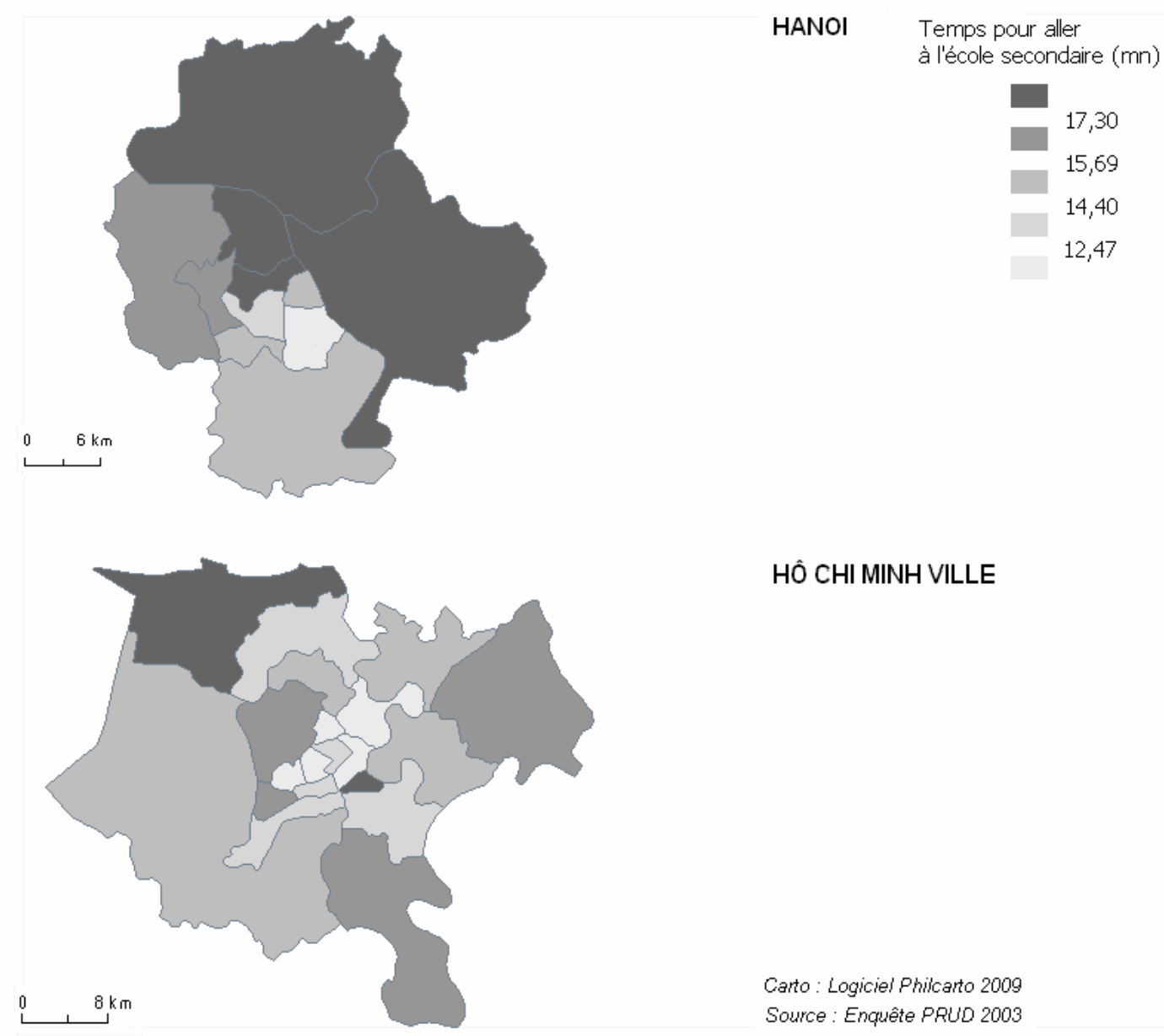

HÔ CHI MINH VILLE

\section{Figure 5 : Temps mis par les élèves de l'école secondaire pour aller à l'école selon l'arrondissement de résidence et la ville (mn)}

\section{Conclusion}

Il existe de multiples relations entre niveau de vie et déplacements dans les métropoles vietnamiennes, mais ces relations sont complexes. Il faut d'abord remarquer que seule une partie de ces relations a été saisie ici du fait que les migrants ruraux-urbains pris en compte parmi la population résidente en ville sont ceux qui ont changé de résidence pour une durée de 6 mois ou plus en venant habiter en ville, selon les définitions internationales et celles du recensement au Viêt-nam; cette population résidente exclut les personnes en déplacement temporaire («visiteurs» en ville venus pour moins de 6 mois), qui effectuent des navettes entre la ville et le village d'origine, où elles sont toujours considérées comme résidentes (il s'agit des « résidents absents »). La spécificité de cette population au Viêt-nam (comme en Chine, mais contrairement à d'autres pays), est qu'elle est en fait venue en ville pour travailler 
tout comme les résidents; elle constitue une "population flottante», en renouvellement permanent, mais en croissance sur le long terme, dont le niveau n'a pour le moment encore jamais été évalué. Nul doute que les «véritables pauvres » en ville, les travailleurs du secteur informel et la population non-enregistrée se trouvent majoritairement en son sein. Une recherche sur ce thème est plus que jamais nécessaire.

Dans le domaine de l'urbanisme, malgré ce qu'on pense parfois, les problèmes des deux métropoles sont largement similaires, mais avec des nuances qui ont été relevées ici.

La « gentrification » du centre-ville, aussi bien à Hô Chi Minh Ville qu'à Hanoi, avec le «glissement» des ménages modestes vers la périphérie, dans un processus de sélection au profit des "riches », pourrait laisser présager pour les " pauvres » un accroissement des distances et des durées pour aller au travail ou pour aller à l'école.

Cependant, parmi ces ménages modestes en zone périurbaine, figurent les agriculteurs des arrondissements périphériques et ruraux, qui se déplacent peu puisqu'ils habitent souvent au milieu de leur parcelle et que leurs enfants fréquentent une école proche de leur domicile. D'autre part, des ménages très aisés rejoignent volontiers la zone périphérique après avoir construit une villa sur une parcelle étendue ou acquis un appartement dans l'un des vastes programmes immobiliers modernes.

Le rôle du permis de résidence, toujours en vigueur, même s'il s'est assoupli, s'exerce de façon similaire : l'absence d'un permis de résidence permanente est un facteur significatif d'augmentation de la pauvreté. Cela se fait surtout sentir en zone périphérique, car en zone rurale, qui compte le plus de "pauvres", les habitants ont en grande majorité un enregistrement permanent.

Au total, les « riches », contrairement à l'attente initiale, ont en fait plus de distance à parcourir et mettent plus de temps pour aller au travail que les « pauvres ». Leurs moyens plus importants leur permettent de recourir à de meilleurs moyens de transport et de choisir leur emploi de manière largement indépendante de sa localisation. Ils sont aussi en mesure de choisir la meilleure école pour leurs enfants, quelle que soit sa distance du domicile.

Les politiques d'assainissement et de déconcentration du centre-ville, dans les deux métropoles, sont « raisonnables », mais avec la «tertiarisation» du centre (remplacement des maisons à usage d'habitation par des immeubles destinés aux services, tels que des bureaux, des banques, des magasins de luxe, de la restauration haut de gamme, etc.), les emplois se développent beaucoup plus vite au centre-ville qu'en périphérie; cela accroît les trajets domicile-travail et pose clairement le problème des infrastructures de tous ordres et du logement de la majorité dans la zone périphérique. En même temps, la multiplication des programmes de construction d'habitations de très haut standing en périphérie, qui sont même hors de portée des couches moyennes de la population, et qui impliquent souvent l'utilisation d'une voiture personnelle pour les déplacements, conduit à s'interroger sur la durabilité de cet urbanisme et sur la possibilité de rentabiliser ces investissements à long terme.

La nécessité d'améliorer et de densifier les transports publics est largement reconnue ; elle reste cependant du domaine du futur, même si le réseau d'autobus a connu des progrès considérables dans les années récentes. Les projets de tramways et de métro existent avec des concours japonais, allemand et français. Mais on peut remarquer que les politiques visant à réduire les embouteillages sont facilement ambivalentes : par exemple, à Hô Chi Minh Ville, 
le projet de péage urbain est susceptible de limiter efficacement les embouteillages comme l'expérience le montre dans d'autres métropoles comme Singapour, mais la construction programmée de grands parkings centraux aura un effet exactement opposé et ce quel que soit le tarif exigé, puisqu'il s'agira bien évidemment de «remplir» ces parkings... Dans ce contexte, le développement d'un réseau dense de transports publics bon marché reste en tout cas plus que jamais un facteur de lutte contre les disparités sociales et d'augmentation de l'égalité des chances dans les métropoles vietnamiennes.

\section{Références bibliographiques}

Central Population and Housing Census Steering Committee, 2009, The 2009 Vietnam population and housing census of 00.00 hours $1^{\text {st }}$ April 2009. Implementation and preliminary result. Hanoi, VIII$48 \mathrm{p}$.

[www.gso.gov.vn/default_en.asp $x$ ?tabid $=615 \& I t e m I D=9268 ; 06 / 01 / 2010]$

Filmer Deon, Pritchett Lant, 1998, Estimating wealth effects without expenditure Data - or Tears: With an application to educational enrolments in States of India. Washington D.C.: The World Bank, 38 p. (World Bank Policy Research Working Paper).

[www.worldbank.org/html/prddr/prdhome/projects/edattain/edtears.pdf; 05/03/2009]

Gubry Patrick, Vu Thi Hông, Lê Van Thanh (Dir.), 2002, Les chemins vers la ville. La migration vers Hô Chi Minh Ville à partir d'une zone du delta du Mékong. Paris : Karthala, CEPED, 343 p. (Hommes et Sociétés).

[http://horizon.documentation.ird.fr/exl-doc/pleins_textes/pleins_textes_7/b_fdi_03_05/010027913.pdf; 03/01/2010]

Gubry Patrick, Lê Thi Huong, Nguyên Thi Thiêng, Pham Thuy Huong, Trân Thi Thanh Thuy, Vu Hoang Ngân (Dir.)/ Nguyễn Thị Thiềng, Lê Thị Hương, Phạm Thuý Hương, Vũ Hoàng Ngân, Trần Thị Thanh Thuỷ, Gubry Patrick (Chủ biên), 2008, Bouger pour vivre mieux. Les mobilités intraurbaines à Hô Chi Minh Ville et Hanoi (Viêt-nam)/ Di chuyển để sống tốt hơn. Di dân nội thị tại Thành Phố Hồ Chí Minh và Hà Nội (Việt Nam). Hanoi : Université nationale d'économie/ Hà Nội : Nhà Xuất Bản Trường Đại Học Kinh Tế Quốc Dân, 293 p. + 278 tr.

[http://horizon.documentation.ird.fr/exl-doc/pleins_textes/divers09-01/010043543.pdf;31/12/2009]

Hardy Andrew, 2001, Rules and Resources: Negotiating the household registration system in Vietnam under reform. Sojourn: Journal of Social Issues in Southeast Asia (Singapore), vol. 16, $\mathrm{n}^{\circ}$ 2, p. $187-212$.

Howe Laura D., Hargreaves James R., Huttly Sharon R.A., 2008, Issues in the construction of wealth indices for the measurement of socio-economic position in low-income countries. Emerging Themes in Epidemiology, 5, 3, 14 p. [www.ete-online.com/content/5/1/3; 05/03/2009]

Pandolfi Laurent, 2001, Une terre sans prix. Réforme foncière et urbanisation au Viêt-nam. Hanoi, 1986-2000. Thèse de doctorat en urbanisme et aménagement, Université de Paris 8, 567 p.

Rutstein Shea Oscar, Johnson Kiersten, 2004, The DHS wealth index. Calverton MD: ORC Macro, ix71 p. (DHS Comparative Reports n ${ }^{\circ} 6$ ).

[www.childinfo.org/files/DHS_Wealth_Index_(DHS_Comparative_Reports).pdf; 05/03/2009]

Villes en Transition Vietnam (VeT), Centre for Sociology and Development Studies Ho Chi Minh City, Institute of Sociology Hanoi, 2005, Impacts of existing residence registration policy on urban poverty alleviation. Two case studies in Hanoi and Ho Chi Minh City. Ho Chi Minh City, 278 p.

Vyas Seema, Kumaranayake Liliani, 2006, How to do (or not to do)... Constructing socio-economic status indices: how to use principal components analysis. Health Policy and Planning, 21(6), p. 459-468.

[http://heapol.oxfordjournals.org/cgi/content/full/21/6/459; 05/03/2009] 

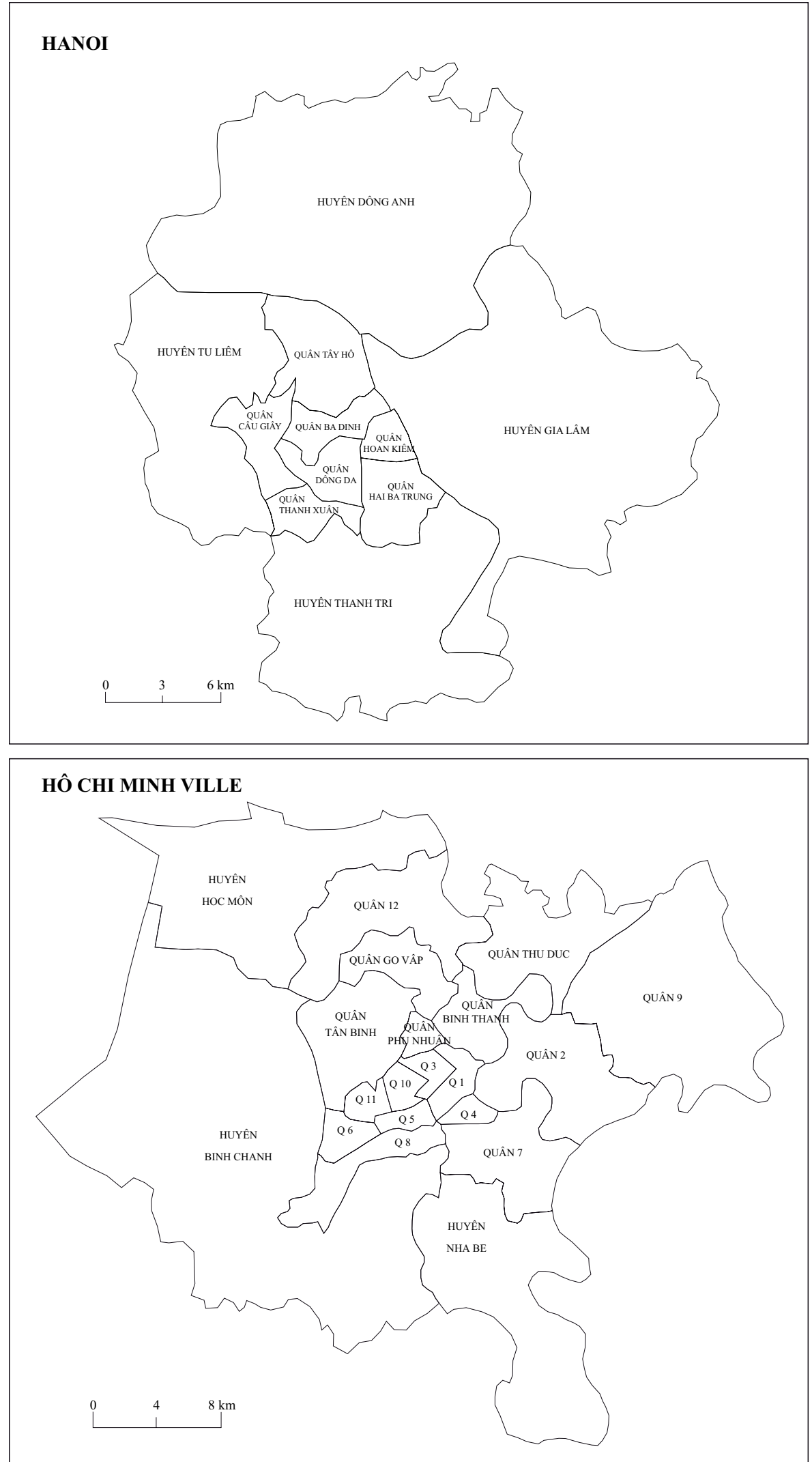

Carte des arrondissements à Hanoi et Hô Chi Minh Ville en 2003 (sauf les arrondissements ruraux de Soc Son à Hanoi, Cu Chi et Cân Gio à Hô Chi Minh Ville) 


\section{Résumé}

Dans le processus de métropolisation en cours dans les grandes agglomérations vietnamiennes, on assiste à un glissement de la population du centre vers la périphérie et à une «gentrification» du centre-ville. Si le cadre de vie s'en trouve généralement amélioré pour ces migrants intra-urbains, les distances à parcourir (par exemple domicile-travail ou domicile-école) augmentent considérablement. Le manque d'infrastructures se fait aussi cruellement sentir dans les zones périphériques nouvellement loties. Le rôle du niveau de vie, mesuré par un « indice de richesse », est mis en lumière. Contrairement à l'attente initiale, ce sont plutôt les " riches » qui ont les trajets les plus longs à parcourir, ce qui leur est permis par les meilleurs moyens de transport dont ils disposent. La lutte contre les inégalités en ville repose immanquablement la question du développement des transports urbains collectifs.

Mots-clés : Migration intra-urbaine, Mobilité temporaire, Niveau de vie, Hô Chi Minh Ville, Hanoi, Viêt-nam

\section{Summary}

\section{Living standards and moves in the Vietnamese metropolises: Ho Chi Minh City and Hanoi}

In the current "metropolisation" process in the large Vietnamese cities, a sliding of the population from centre towards suburbs and a "gentrification" of the city-centre appear. While living environment is generally improved for these intra-urban migrants, the distances to be covered (for example from home to work or from home to school) increase considerably. The lack of infrastructures is also desperately felt in the recently built peripheral zones. The role of living standards, measured by a "wealth index", is emphasized. Contrary to the initial expectation, there are rather "rich people" which have the longest routes to be gone through, what is enabled by their better transportation means. The fight against disparities in the city still inevitably arises the question of the development of public transportation means.

Keywords: Intra-urban migration, Temporary move, Living standards, Ho Chi Minh City, Hanoi, Vietnam 